\title{
Segment 8 hepatic vein reconstruction in a living donor after left hepatectomy
}

\author{
Chih-Yi Chen, Chao-Long Chen, Chee-Chien Yong, Aldwin D. Ong \\ Liver Transplantation Center, Department of Surgery, Kaohsiung Chang Gung Memorial Hospital, Kaohsiung, Taiwan, China \\ Correspondence to: Chao-Long Chen, MD, PhD (Hon). Liver Transplantation Center, Department of Surgery, Kaohsiung Chang Gung Memorial \\ Hospital, 123 Ta-Pei Road, Niao Sung, Kaohsiung 833, Taiwan, China. Email: clchen@cgmh.org.tw.
}

Submitted May 06, 2021. Accepted for publication May 18, 2021.

doi: $10.21037 / \mathrm{hbsn}-21-179$

View this article at: http://dx.doi.org/10.21037/hbsn-21-179

Liver transplantation (LT) has been accepted worldwide as standard of treatment for end-stage liver disease. Due to expanding indications for LT and shortage of cadaveric donors, living donor liver transplantation (LDLT) has emerged as an important alternative to augment the donor pool (1). Justifiably, donor safety is the fundamental priority in LDLT.

Donor safety is ensured by adequate remnant liver volume with good perfusion and drainage. Delineation of hepatic venous anatomy by preoperative imaging is crucial for surgical planning. Insufficient hepatic venous outflow could result in severe congestion with subsequent liver dysfunction. Therefore, venous outflow must be meticulously secured in both the donor and the recipient (2-4). We herein report a successful case of a left liver donor with a significant segment 8 hepatic vein (V8) reconstructed with a ringed polytetrafluoroethylene (PTFE) graft in order to alleviate extensive congestion in the remnant anterior segment of the donor remnant liver.

The donor was a 37-year-old man, cousin of the recipient who was diagnosed with recurrent hepatocellular carcinoma in the remnant liver two years after undergoing right hepatectomy. The donor had a body mass index of $18.6 \mathrm{~kg} / \mathrm{m}^{2}$. He had no significant medical history, and his clinical and laboratory examinations were unremarkable. Computed tomography (CT) angiography revealed a calculated liver volume of $1,267 \mathrm{~mL}$, with the left liver being $522 \mathrm{~mL}$, consisting $41.2 \%$ of the total liver volume. Preoperative congestive volume studies were not done, but assessment for congestion was intended intraoperatively with test clamping. Contraction of the recipient's right hepatic fossa from previous right hepatectomy precluded the feasibility of a right liver donation. However, the donor had a middle hepatic vein (MHV)-dominant right liver, with a small right hepatic vein (RHV) and a significant V8 outflow (Figure 1).

The recipient did not have other donor-compatible candidates in his family and had a low chance of receiving a cadaveric liver given a model for end-stage liver disease (MELD) score of 7 points. After understanding the risks, benefits and contingencies of the procedure, with emphasis on possible donor hepatic venous reconstruction to optimize outflow, both the donor and the recipient expressed their strong desire to proceed with LDLT. Informed consent was obtained from both parties.

During transection of the liver parenchyma, the exposed V8 measured $8 \mathrm{~mm}$ (Figure 2A). Test clamping of V8 resulted in an unexpectedly large demarcated area of congestion in the remnant right liver (Figure 2B). The V8 tributary was subsequently tagged for reconstruction before being divided. After graft procurement, a ringed PTFE interposition graft $(8 \mathrm{~mm} \times 50 \mathrm{~mm})$ was anastomosed in place as conduit between the V8 stump of the remnant liver and the IVC (Figure 2C). Upon release of the V8 bulldog clamp, the congested area immediately resumed a pinkish coloration (Figure 2D). Intraoperative Doppler ultrasound confirmed its patency, as well as biphasic V8 waveform. Since the congestion of the entire anterior segment resolved (Figure 2D), reconstruction of V5 was deemed to be unnecessary. All successive surgical steps of left liver graft procurement were routine (5).

The left liver graft, comprising segments 1 to 4 and the MHV, weighed 516 grams and had a graft-to-recipient weight ratio of $0.67 \%$. The donor's remnant liver was 

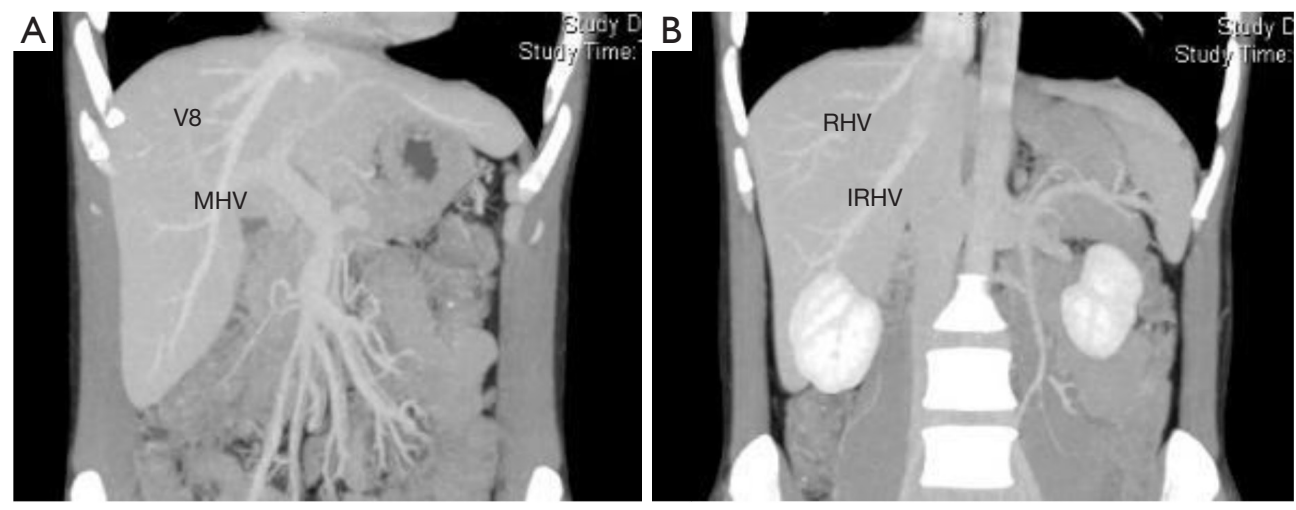

Figure 1 Donor CT angiography showing (A) an MHV-dominant right liver with a significant V8, and (B) a relatively small RHV. CT, computed tomography; MHV, middle hepatic vein; RHV, right hepatic vein; IRHV, inferior right hepatic vein.
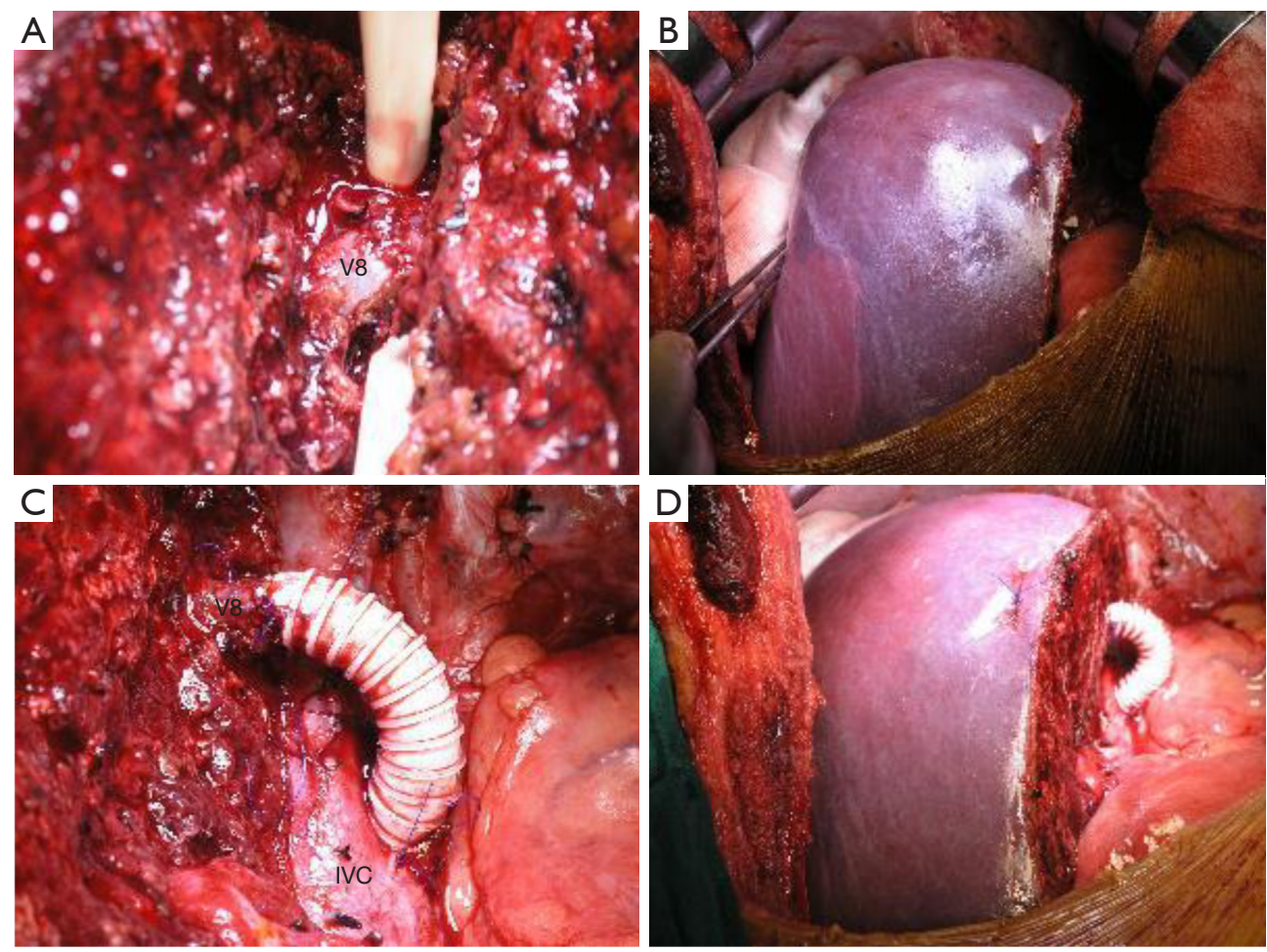

Figure 2 Intraoperative findings: (A) isolated V8 measuring $8 \mathrm{~mm}$; (B) large demarcated area of congestion after test clamping; (C) ringed PTFE interposition graft between the V8 stump and the IVC; (D) almost immediate disappearance of congestion after V8 reconstruction. PTFE, polytetrafluoroethylene; IVC, inferior vena cava.

\section{$59.3 \%$ of his estimated total liver volume.}

The postoperative courses of both the donor and the recipient were uneventful. The donor received intravenous heparin infusion (100 U/kg/day) for two weeks. Doppler ultrasound on postoperative days 1, 4, 13, and 90 showed consistent patency and functionality of the reconstructed
V8-IVC conduit. CT angiography at three months showed patent outflow of V8, RHV, and IRHV, with the remnant right liver regenerating to $972 \mathrm{~cm}^{3}$. CT angiography at 6 months showed patency of the reconstructed V8 and PTFE interposition graft (Figure 3). Six years after LDLT, both the donor and the recipient are in good clinical health 


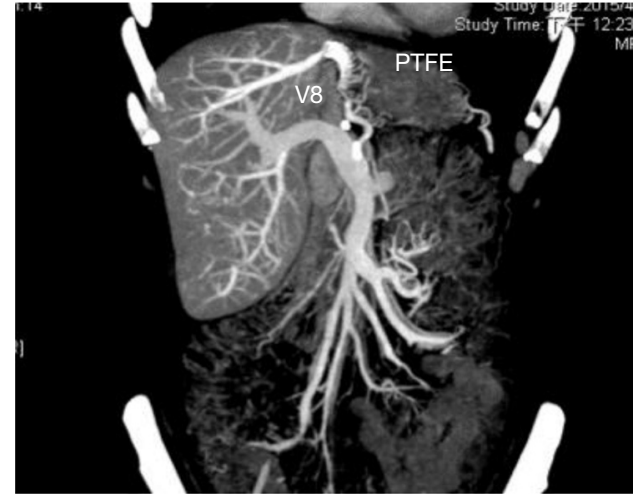

Figure 3 Donor CT angiography 6 months after donation shows a patent V8-PTFE graft outflow. CT, computed tomography; PTFE, polytetrafluoroethylene.

with sustained venous patency.

LDLT has proven to be a highly efficacious alternative to deceased donor LT (1). In our center, LDLT constitutes $88 \%$ of all performed LTs. The primary goal of LDLT is to optimize recipient outcome without compromising donor safety. Preoperative mapping and identification of liver anatomy are critical for donor selection and surgical planning. Insufficient hepatic venous outflow is a serious technical complication for both donor and recipient; a resulting hepatic congestion may lead to a highly morbid sequelae and even mortality $(2,3)$.

Hepatic venous outflow reconstruction with an interposition graft has been adopted widely for right liver recipients without $\mathrm{MHV}$ drainage to alleviate hepatic venous congestion in the right anterior segment $(6,7)$. Reconstruction of MHV tributaries was previously accomplished using autologous or homologous vascular grafts $(7,8)$. In countries where deceased organ donation is scarce, the relative undersupply of vascular allografts has sparked an interest in the utility of artificial grafts as an alternative (7). Hwang et al. demonstrated satisfactory results for MHV reconstruction using PTFE grafts, with high patency rates comparable to that of iliac vein grafts (7). Yi et al. has likewise reported the utility of PTFE as interposition graft for V5 and V8 outflow reconstruction in the recipient (9). Complications associated with PTFE used for right liver graft MHV reconstruction, including infection and migration into the gastrointestinal tract, was observed to be $0.5 \%$ (10). In our center, both cryopreserved homologous vascular grafts and synthetic grafts for MHV tributary reconstruction, when indicated, have routinely been used in recipients; no PTFE-related infections or migration have been encountered in all cases. A cryopreserved homologous graft was not considered for the donor in order to mitigate the risk of infection or an allogenic immune reaction; on the other hand, autologous venous grafts, such as the internal jugular vein, would have entailed unnecessary additional surgery.

In the LDLT case presented, the recipient could only accommodate a left liver graft due to a contracted right hepatic fossa as a result of previous right hepatectomy. His only suitable donor however, had an MHV-dominant right liver, with a small RHV and a significant V8 tributary. Ligating this V8 tributary during donor left hepatectomy resulted in congestion of the remnant liver due to a compromised venous drainage from the right anterior segment. Although previous studies have shown that up to $40-70 \%$ of liver function of the congested liver volume may be preserved $(11,12)$, decision was made to reconstruct the V8 drainage in the donor based on our extensive experience in the use of interposition grafts in recipient outflow reconstruction, in order to maximally preserve remnant liver function. Repeated imaging studies confirmed the patency of the reconstructed hepatic venous outflow. To the best of our knowledge, this is the first published description of a successful anterior segment outflow reconstruction using a PTFE graft in a left liver living donor presenting with an MHV-dominant right liver.

In LDLT, donor safety is of utmost importance. Donor risk must always be prudently balanced with recipient benefit. For left liver donors with an MHV-dominant right liver, the increased donor risk from potentially needed outflow reconstruction must always be extensively discussed with both donor and recipient. Proper donor selection, extensive preoperative planning, and experience-guided intraoperative decision-making are of utmost importance to optimize and preserve donor remnant volume and function. This risk varies significantly with surgeon and institutional experience in recipient graft outflow reconstruction. Out of over 1,800 cases of LDLT performed in our center, this was in fact the first donor in which V8 reconstruction was judged to be necessary.

Reporting innovations in surgical technique, particularly those tackling complex liver anatomy, is important as it is part of finding secure ways to further safely expand the organ donor pool. Donor anterior segment outflow reconstruction, based on extensive LDLT experience, detailed preoperative imaging, and precise surgical planning, can be performed safely. 


\section{Acknowledgments}

Funding: None.

\section{Footnote}

Provenance and Peer Review: This article was a standard submission to the Hepatobiliary Surgery and Nutrition. The article has undergone external peer review.

Conflicts of Interest: All authors have completed the ICMJE uniform disclosure form (available at https://hbsn. amegroups.com/article/view/10.21037/hbsn-21-179/coif). Dr. CLC serves as an unpaid editorial board member of Hepatobiliary Surgery and Nutrition. The other authors have no conflicts of interest to declare.

Ethical Statement: The authors are accountable for all aspects of the work in ensuring that questions related to the accuracy or integrity of any part of the work are appropriately investigated and resolved. Written informed consent was obtained from the patient for publication of this manuscript and any accompanying images.

Open Access Statement: This is an Open Access article distributed in accordance with the Creative Commons Attribution-NonCommercial-NoDerivs 4.0 International License (CC BY-NC-ND 4.0), which permits the noncommercial replication and distribution of the article with the strict proviso that no changes or edits are made and the original work is properly cited (including links to both the formal publication through the relevant DOI and the license). See: https://creativecommons.org/licenses/by-nc-nd/4.0/.

\section{References}

1. Chen CL, Kabiling CS, Concejero AM. Why does living donor liver transplantation flourish in Asia? Nat Rev Gastroenterol Hepatol 2013;10:746-51.

2. Uchida K, Taniguchi M, Shimamura T, et al. Three-

Cite this article as: Chen $\mathrm{CY}$, Chen CL, Yong CC, Ong AD. Segment 8 hepatic vein reconstruction in a living donor after left hepatectomy. HepatoBiliary Surg Nutr 2021;10(4):579-582. doi: 10.21037/hbsn-21-179 dimensional computed tomography scan analysis of hepatic vasculatures in the donor liver for living donor liver transplantation. Liver Transpl 2010;16:1062-8.

3. Lee S, Park K, Hwang S, et al. Anterior segment congestion of a right liver lobe graft in living-donor liver transplantation and strategy to prevent congestion. J Hepatobiliary Pancreat Surg 2003;10:16-25.

4. de Villa VH, Chen CL, Chen YS, et al. Right lobe living donor liver transplantation-addressing the middle hepatic vein controversy. Ann Surg 2003;238:275-82.

5. Chen CL, Chen YS, de Villa VH, et al. Minimal blood loss living donor hepatectomy. Transplantation 2000;69:2580-6.

6. Lee SG. A complete treatment of adult living donor liver transplantation: a review of surgical technique and current challenges to expand indication of patients. Am J Transplant 2015;15:17-38.

7. Hwang S, Jung DH, Ha TY, et al. Usability of ringed polytetrafluoroethylene grafts for middle hepatic vein reconstruction during living donor liver transplantation. Liver Transpl 2012;18:955-65.

8. Sugawara Y, Makuuchi M, Akamatsu N, et al. Refinement of venous reconstruction using cryopreserved veins in right liver grafts. Liver Transpl 2004;10:541-7.

9. Yi NJ, Suh KS, Lee HW, et al. An artificial vascular graft is a useful interpositional material for drainage of the right anterior section in living donor liver transplantation. Liver Transpl 2007;13:1159-67.

10. Ha TY, Hwang S, Jung DH, et al. Complications analysis of polytetrafluoroethylene grafts used for middle hepatic vein reconstruction in living-donor liver transplantation. Transplant Proc 2014;46:845-9.

11. Kawaguchi Y, Ishizawa T, Miyata Y, et al. Portal uptake function in veno-occlusive regions evaluated by real-time fluorescent imaging using indocyanine green. J Hepatol 2013;58:247-53.

12. Ninomiya M, Shirabe K, Kayashima H, et al. Functional assessment of the liver with gadolinium-ethoxybenzyldiethylenetriamine penta-acetate-enhanced MRI in livingdonor liver transplantation. Br J Surg 2015;102:944-51. 